\title{
Derivative estimation of triangular patch by using cubic least square method
}

\begin{abstract}
Smooth surface reconstruction of scattered data built from Delaunay triangulation need the partial derivatives to be defined at the vertices of the triangles for all the data points. Partial derivatives at the vertices of triangular elements and at the midpoint of each side usually are unavailable therefore it is a needed to approximate the derivatives information at the vertices and at the midpoint of each side. The common method use to estimate partial derivatives was the quadratic approximation of least square method. This research focused to estimate partial derivatives by using cubic approximation of least square method and compare the surface obtained between quadratic and cubic approximation. The research also implement the use of interpolating surface of cubic Bezier triangular patch. The result of the study shows comparison of interpolating surface produced by different type of functions for quadratic and cubic least square. The maximum and minimum error was also calculated and maximum error between quadratic and cubic least square was generated when using saddle function while the minimum error is on the exponential function.
\end{abstract}

Keyword: Least square method; Cubic; Triangular patch 